\title{
Eye Position Effect on Audio-Visual Fusion Involves New Proposals for Multimodal Interface Design
}

\author{
David Hartnage, Alain Bichot, Patrick Sandor, and Corinne Roumes \\ Département Action \& Cognition en Situation Opérationnelle, \\ Institut de Recherche Biomédicale des Armées, BP 73, \\ 91223 Brétigny-sur-Orge, France \\ dhartnagel@imassa.fr
}

\begin{abstract}
Combination of audio and visual information is expected to ensure an efficient interface design for spatial information. Then, we focus on Audiovisual (AV) fusion referred to the perception of unity of audio and visual information despite there spatial disparity [1]. Previous experiment showed that AV fusion varied over space mainly with horizontal eccentricity [2]. As audio spatial information is coded in relation to head position and visual information is coded relative to eye position, question arises on eye position effect. The current psychophysical experiment investigates the effect of horizontal eye position shift on the variation of AV fusion over the 2D frontal space. Results showed that eye position affects AV fusion. Current data support the need for including eye position inputs when displaying redundant visual and auditory information in integrated multimodal interfaces. Results are discussed considering the probable effect of visual display structure.
\end{abstract}

\section{Introduction}

A wide variety of working situations such as teleoperation, simulation for training and air operations, require the use of a virtual 3D restitution of the world. The so-called Virtual Reality can remain partial in the case of Augmented Reality when computed data are displayed overlaying the natural perception of the environment. The HumanMachine Interface (HMI) necessarily provides the operator with information in a non natural way. The human has to deal with a new kind of reality characterized by new physical rules. In order to design more ecologically the interface and to ensure the expected level of efficiency, it is worth understanding how the human assumes these new realities. A present HMI design challenge concerns multisensory integration. Combining different sensory information provides faster reaction time [3] and ensures a redundant information processing. These benefits are decisive in time determinant situations and may enhance situation awareness. This defy implies to study the way human perceive auditory and visual information as coming from the same location in space. This phenomenon is referred to as audio-visual (AV) fusion. The present paper concerns the rules of AV fusion in space and their involvement for HMI design.

Perception of the world is basically multimodal; the world is not spread over sensory cues, it is perceived as unified [4]. Experimental results have shown an enhancement of cell responses for multimodal stimuli in various locations of the 
nervous system [5][6]. Association of sensory cues provides many advantages: it is ecological, accurate, efficient and reliable; information is redundant; and responses are faster. Information coming from different modalities may be perceived as one. This phenomenon of perceptual fusion is usually compared to the ventriloquism effect [7]: the perception of the spatial location of a sound is biased in the direction of a visual stimulus (visual capture). Audio-visual fusion (AV fusion) has been investigated by Godfroy et al. [2]. They showed that AV fusion capability varied with the eccentricity of the bimodal stimulation in the participant's perceptive field, the smallest spatial windows laying in the median sagittal plane. AV spatial windows were found to be symmetrical in relation to that plane. So, AV fusion space was considered as head-centered. These variations over space followed closely the spatial resolution of audition. There are quite a large number of reference frames depending on the sensory system involved, the context, and the goal [8]. Each of them can be egocentric (relative to the subject), allocentric (relative to the environment) or even geocentric (relative to the earth). What kind of frame of reference supports the AV fusion in space? Actually, primary unimodal egocentric reference frames are different whether information involves the visual sensory system or the auditory system. As vision information is initially coded relative to the retina, which depends on the position of the eye, the reference frame for vision is considered as eye-centered. For audition, spatial information depends mainly on inter-aural time differences (ITD), inter-aural level differences (ILD) in azimuth, and spectral cues in elevation [9]. These cues depend on the position of the head; so, the auditory reference frame is considered as head-centered.

The aim is to define the limits of human AV fusion perception when the eyes are shifted in order to display trustworthy bimodal information whenever the operator does not look straight ahead, which may be the most common situation. Results will be discussed in the frame of the suggested applications.

\section{Methods}

\subsection{Subjects}

Seven volunteers participated in this study, 4 women and 3 men aged, from 25 to 45 . They all had normal or corrected to normal vision and no auditory defects.

\subsection{Experimental Setup}

\section{Stimuli Control}

The subject was located at the center of an acoustically-transparent, hemi-cylindrical screen, $120 \mathrm{~cm}$ in radius and $145 \mathrm{~cm}$ in height. The subject's head was maintained by a custom bite-board with the eyes at a $110-\mathrm{cm}$ height. The head was oriented $10^{\circ}$ leftward of the center of the screen to increase the space of investigation when a fixation spot was presented $20^{\circ}$ to the right. The orientation of the gaze was monitored with an ASL $504(50 \mathrm{~Hz})$ eye-tracker placed $45 \mathrm{~cm}$ in front of the subject at a level lower than the field of investigation to prevent from any visual masking. To 
avoid any effect of allocentric cues, the experimental room was in total darkness, and noise level was reduced as much as possible $\left(<39 \mathrm{~dB}_{\mathrm{A}}\right)$.

Stimuli could only be presented if the subject was looking at the red fixation spot with an angular error less than $1.66^{\circ}$ for a mean duration of $500 \mathrm{~ms}(300-700 \mathrm{~ms})$. Such a feedback between the eye-tracker sampling and the experimental software was used guarantee the dissociation of the two references frames at the bimodal stimulus onset. The bite-board permitted the control of the head position (i.e. the auditory reference frame) and the eye-tracker permitted the control of the eye position (i.e. the visual reference frame).

The visual part of the bimodal $500 \mathrm{~ms}$ stimulus was a $1^{\circ}$ spot of light, it was provided by a green laser beam (MELLES-GRIOT, $532 \mathrm{~nm}, 5 \mathrm{~mW}$ ) attenuated with optical filters in order to reduce luminance at $3 \mathrm{~cd} . \mathrm{m}-2(<5 \mu \mathrm{W})$.

The auditory part of the bimodal stimulus was a $49 \mathrm{dBA}$ broad-band pink noise delivered by one of the 19 loudspeakers (LS) located behind the screen, oriented toward the subject's head.

\section{Disparity}

For each of the 7 loudspeakers at eye level, 61 disparities between the spot and the sound source were tested. The spot could be displayed $0^{\circ}$ to $20^{\circ}$ horizontally apart from the center of the associated loudspeaker. At $0^{\circ}$ of azimuth the spot could be displayed $0^{\circ}$ to $30^{\circ}$ apart with a $5^{\circ}$ step in elevation.

For all other loudspeakers only 9 disparities were tested.

\subsection{Task}

The subject had to judge the perception of unity emerging from the bimodal stimulus using a joystick. When the spot and the pink noise were perceived as coming from a unique and common location in space, "fusion" response was selected by pulling the joystick. When the two unimodal stimuli were perceived as issuing from two distinct locations, the "non fusion" response was selected by pushing the joystick.

\section{Results}

For most of the loudspeakers, fusion limits in elevation could not be established. Subjects still responded "fusion" for more than $50 \%$ of the trials for disparity up to $30^{\circ}$.

AV fusion limits in azimuth varied along the horizontal dimension in the perceptive field $\left(\mathrm{F}_{(6,1243)}=21.783 ; \mathrm{p}<.001\right)$. This variation appeared laterally deviated in the direction of eye shift in the reference frames dissociated condition. No significant difference was obtained between the 2 conditions $\left(\mathrm{F}_{(1,1243)}=0.973\right.$; ns $)$ but the interaction between conditions and eccentricity was significant $\left(\mathrm{F}_{(6,1243)}=4.93\right.$; $\mathrm{p}<.05)$. The signs of the differences were analyzed. Differences between the two conditions were significant for 5 paired eccentricities out of the 7 tested. Moreover, the sign of the differences changed as if the curve of the dissociated condition would have been shifted $10^{\circ}$ to the right (toward eye shift, 50\%). 


\section{Discussion}

The present basic experiment was conducted in order to study the effect of gaze shift on AV fusion. Results show that lateral gaze shift influences AV fusion perception in azimuth but not in elevation. Data collected in this experiment must be taken into account when considering HMI design. Designers from both virtual environments and enhanced perception used head tracker, and the displayed data changes in relation to head movement. As a consequence, gaze is assumed to be in line with head position when displaying visual and auditory information. It can be suggested currently that the lack of eye monitoring may bias the expected perception of visual and auditory redundant information.

This question is particularly important for aircraft Head Mounted Display's (HMD), where time is determinant. In such a 3D dynamic environment where the relative position of the various actors may dramatically change, it may be fruitful for the pilot to take advantage of the high mobility of the eyes compared to the dynamic of the head equipped with helmet. Indeed, the spherical shape of the eye, it's location in the hemispherical socket, make any change in the eye orientation effortless. So it can be inferred that pilots usually favor eye movement instead of head movement. In many situations, especially in combat, there are high $\mathrm{Gz}$ accelerations, head movement will be limited and pilots will use more eye movement.

Even though, it can be argued that this situation is very complex compared with psychophysics tasks, the present results have to be considered to avoid error in multimodal display.

The experiment reported in this paper deliberately involved non informative visual and auditory stimuli. No allocentric cues were available. The bimodal stimulus was the only sensory input to process. Such conditions are in crucial opposition with HMIs where a high flow of data is concerned. Nevertheless, such basic data are not available in the literature.

The ideal technological solution would take into account head and eye position to change the display in relation to the position of the eye and head to match with the perceptual space of the operator.

To overpass this technical difficulty, it may be easier to exchange a hardware refinement for a software manipulation. If AV fusion naturally varies according to head and eye orientation, can an additional item in the information displayed provide a counter-measure to the eye shift effect?

The present experiment was conducted in total darkness, with no additional visual cue provided. However, previous experiments have shown that visual display can influence AV fusion perception as well. Perhaps visual cues like linear symbols enhancing the edges of the display or a peculiar spatial arrangement of the features structuring visual space can give an allocentric reference frame to the subject. This allocentric reference frame would compensate for dissociation between auditory and visual reference frames. These data suggested further investigations to determine whether visual or auditory additional markers in the perceptive field may counteract eye inputs effect on audio-visual fusion. Moreover, such selected visual and auditory stimuli may also counterbalance the reported tendency of an upward offset of the perceived location of auditory sources in darkness [10]. 


\section{References}

1. Bertelson, P., Radeau, M.: Cross-modal bias and perceptual fusion with auditory-visual spatial discordance. Perception \& psychophysics 29(6), 578-584 (1981)

2. Godfroy, M., Roumes, C., Dauchy, P.: Spatial variations of visual-auditory fusion areas. Perception 32(10), 1233-1245 (2003)

3. Diederich, A.: Intersensory facilitation, vol. 369. Peter Lang, Frankfurt (1992)

4. Gibson, J.J.: The senses considered as perceptual systems. Greenwood Press, Westport (1966)

5. Stein, B.E., Meredith, A.M.: The merging of the senses. The MIT press, Cambridge (1993)

6. Giard, M.-H., Peronnet, F.: Visual-auditory Integration during Multimodal Object recognition in Humans: A Behavioral and Electrophysiological Study. Journal of Cognitive Neuroscience 11(5), 473-490 (1999)

7. Jack, C.E., Thurlow, W.R.: Effect of degree of visual association and angle of displacement on the ventriloquism effect. Perceptual and Motor Skills 37, 967-979 (1973)

8. Paillard, J.: Motor and representational framing of space. In: Paillard, J. (ed.) Brain and space, pp. 163-182. Oxford University Press, Oxford (1991)

9. Blauert, J.: Spatial Hearing. In: The psychophysics of human sound localization, The MIT press, London (1983)

10. Pedersen, J.A., Jorgensen, T.: Localization performance of real and virtual sound sources. In: New Directions For Improving Audio Effectiveness, pp. 29.1-29.30. RTO, France (2005)

11. Perrot, D.R., Saberi, K.: Minimum audible angle thresholds for sources varying in both elevation and azimuth. Journal of the Acoustical Society of America 87, 1728-1731 (1990)

12. Witkin, H.A., Asch, S.E.: Studies in space orientation. IV. Further experiments on perception of the upright with displaced visual fields. Journal of Experimental Psychology 38, 762-782 (1948) 